\title{
Os efeitos da expansão rápida da maxila sobre a permeabilidade nasal avaliados por rinomanometria e rinometria acústica
}

Bruno Alberto Vidotti*, Inge Elly Kiemle Trindade**

\begin{abstract}
Resumo
O objetivo deste artigo foi analisar os achados da literatura acerca das repercussões da expansão rápida da maxila (ERM) sobre a permeabilidade nasal, pela rinomanometria, que avalia a resistência oferecida pela cavidade nasal à passagem do fluxo aéreo, e pela rinometria acústica, que mede as áreas seccionais e os volumes de diferentes segmentos da cavidade nasal. Estudos que utilizaram a rinomanometria evidenciaram que a ERM determina uma diminuição significativa da resistência nasal, assim como o aumento da área de secção transversa mínima nasal (ASTM) em parcela significativa de pacientes. Estudos com rinometria acústica mostraram que a ERM determina, adicionalmente, um aumento significativo do volume da cavidade nasal anterior. Entretanto, é consenso que a ERM não se justifica, por si, como forma de induzir um padrão nasal de respiração em respiradores bucais habituais, apesar dos benefícios trazidos pela ERM em termos de permeabilidade nasal.
\end{abstract}

Palavras-chave: Técnica de expansão palatina. Rinomanometria. Rinometria acústica.

\section{INTRODUÇÃO}

A expansão rápida da maxila (ERM) é um procedimento ortodôntico e ortopédico que visa a correção da atresia real ou relativa da maxila, por meio de diferentes tipos de aparelhos disjunto$\operatorname{res}^{6,7}$. Sua ação ortopédica se dá, basicamente, pela abertura da sutura palatina mediana, o que garante ganho real de tecido ósseo na dimensão transversal da maxila, e tem o efeito potencial de alterar as dimensões internas da cavidade nasal ${ }^{13,14,20}$. Este efeito é de particular importância porque, por um lado, evidências mostram que a obstrução nasal pode levar à atresia do arco maxilar ${ }^{19,34}$; por outro, a atresia maxilar pode determinar um decréscimo da largura da cavidade nasal, o que teoricamente compromete a permeabilidade nasal, ou seja, a passagem do ar através da cavidade nasal, podendo levar à respiração bucal de suplência. Tomando por base este raciocínio, alguns autores passaram a sugerir o uso da ERM como forma de tratamento da obstrução nasal ${ }^{13,38}$.

Os primeiros estudos ${ }^{13,14,38}$ que avaliaram os efeitos da ERM sobre a cavidade nasal basearamse apenas em observações clínicas subjetivas ou

* Cirurgião-dentista pela Faculdade de Odontologia de Bauru-USP

** Professora titular do Departamento de Ciências Biológicas da Faculdade de Odontologia de Bauru-USP e do Laboratório de Fisiologia do Hospital de Reabilitação de Anomalias Craniofaciais (HRAC-USP). 
em medidas radiográficas, inadequadas para avaliar as modificações tridimensionais induzidas pela expansão. Buscou-se, então, a utilização de métodos que permitissem uma avaliação mais objetiva das variações da permeabilidade nasal em resposta à ERM. Com este propósito, alguns autores ${ }^{16,17,24,29}$ passaram a utilizar a rinomanometria, um teste dinâmico baseado em princípios fisiológicos, que mede a resistência oferecida pela cavidade nasal à passagem do fluxo aéreo. Mais recentemente, um novo recurso passou a ser utilizado $3,10,15,22$ : trata-se da rinometria acústica, um teste estático, baseado em princípios acústicos, que permite a avaliação de áreas seccionais e de volumes de diferentes segmentos da cavidade nasal.

O presente trabalho teve por objetivo revisar os estudos descritos na literatura que utilizaram a rinomanometria e a rinometria acústica para avaliar os efeitos da ERM sobre a geometria nasal, verificando a aplicação dada a essas técnicas e seus principais resultados. Pretendeu-se, desta forma, colher informações que possibilitem uma melhor compreensão do real efeito da ERM sobre a função naso-respiratória, com base em dados objetivos de diagnóstico, e verificar se a ERM por si só é um procedimento recomendado para o tratamento da obstrução nasal.

\section{REVISÃO DE LITERATURA}

A ERM, como forma de tratamento da atresia da maxila, foi introduzida por Angell ${ }^{1}$, em 1860, ao descrever um aparelho com parafuso expansor para promover a separação dos ossos maxilares, mas sua efetividade passou a ser amplamente reconhecida com os trabalhos de Hass, o primeiro deles ${ }^{13}$ publicado em 1965. A partir de então, a técnica vem sendo sistematicamente utilizada, não só como tratamento de eleição para a correção da deficiência transversal da maxila, mas também como procedimento adjunto na tração reversa da maxila em casos de Classe III esquelética, com pequenas variações quanto ao tipo de aparelho, tipo de ativação, tempo de contenção, entre outros as- pectos $^{2,5,6,7,8,11,26,27}$.

É vasta a literatura quanto aos efeitos provocados pela ERM, no que se refere, especificamente, às modificações esqueléticas. Estudos ${ }^{14,20}$ da década de 70 demonstraram - por imagens radiográficas - que a abertura da sutura palatina mediana, em vista frontal, se dá de forma triangular, com vértice voltado para a cavidade nasal; e que, em uma visão transversal, se dá também de forma triangular, com a base maior voltada para a espinha nasal anterior. $\mathrm{O}$ movimento transversal das paredes laterais da cavidade nasal, e o conseqüente afastamento das conchas nasais em relação ao septo nasal, também foi observado, o que levou alguns autores $^{14,38}$ a incluir a melhora da respiração nasal entre os efeitos benéficos da ERM. Contudo, para chegar a esta conclusão, esses autores basearam-se em relatos subjetivos da permeabilidade nasal e na análise radiográfica da cavidade nasal, que apenas fornece informação de caráter bidimensional. Dentro desse contexto, fazia-se necessário o uso de métodos mais objetivos de avaliação da permeabilidade nasal após a ERM, o que veio a ser possível com o emprego, por diversos autores, da rinomanometria ${ }^{16,17,24,29,37}$ e, mais recentemente, da rinometria acústica ${ }^{3,10,15,22}$.

Em termos funcionais, o método que melhor permite caracterizar a permeabilidade nasal é a rinomanometria, que envolve a medida simultânea da diferença de pressão gerada entre a extremidade anterior e a extremidade posterior da cavidade nasal e o fluxo aéreo através do nariz na respiração de repouso (Fig. 1). Com base no quociente entre essas variáveis (pressão/fluxo), a resistência nasal $(\mathrm{RN})$ é calculada ${ }^{31,35}$. Alternativamente, é possível calcular a área de secção transversa mínima da via nasal (ASTM) ${ }^{31,35}$, que tem a vantagem de ser uma medida fluxo-independente, ou seja, é a mesma em qualquer fluxo respiratório, ao contrário da $\mathrm{RN}$, que varia dependendo do esforço respiratório do paciente. Vale ressaltar que a rinomanometria não fornece a localização exata do ponto de maior constrição (menor área seccional) da cavidade nasal. 


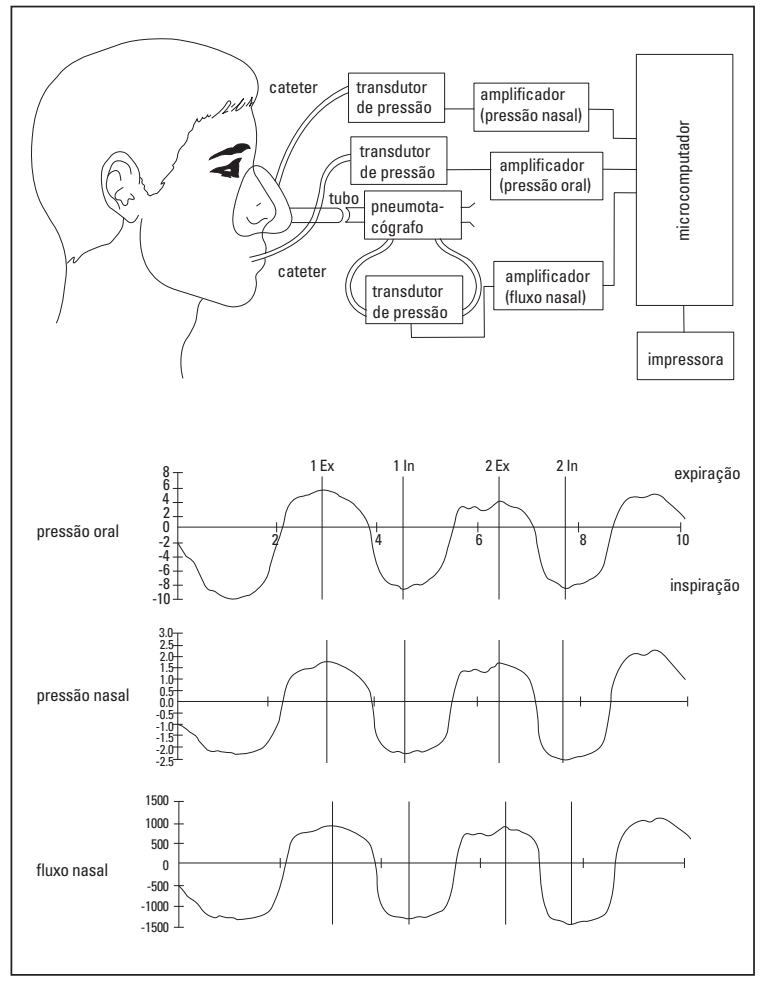

FIGURA 1 - Esquema representativo da rinomanometria e traçados de pressão oral, pressão nasal e fluxo nasal em adulto normal em repouso (Adaptado de TRINDADE e TRINDADE JUNIOR ${ }^{32}$.

Valores de RN e ASTM foram determinados em crianças e adultos com nariz normal ou obstruí$\mathrm{do}^{35,36}$. Estudos realizados no Brasil estabeleceram valores de ASTM para a população local ${ }^{31,32}$.

Os trabalhos que investigaram os efeitos da ERM com a rinomanometria, utilizando a $\mathrm{RN}$ como a variável de estudo, de modo geral, avaliaram pacientes com idade entre 7 e 22 anos, comparativamente a grupos controle compostos por indivíduos da mesma faixa etária sem obstrução nasal e não submetidos à ERM ${ }^{16,17,24,29}$. Nesses estudos, a RN foi avaliada antes da expansão ${ }^{16,17,24,29}$, logo após o período de ativação ${ }^{16,17,24}$, após o período de contenção $0^{17,24,29}$ e 1 ano após a expansão ${ }^{16}$. Em todos, observou-se redução da RN após a ERM, da ordem de 30 a 40\%, que se mostrou estável após o período de contenção ${ }^{17,24,29}$ e mesmo após 1 ano da expansão ${ }^{16}$. As maiores reduções da $\mathrm{RN}$ não foram necessariamente associadas às maiores expansões ${ }^{29}$, mas sim à presença de uma RN elevada antes da expansão ${ }^{16,17,29}$. Esses achados levaram alguns autores ${ }^{16,29}$ a desconsiderar o uso da ERM para fins exclusivos de correção dos problemas respiratórios, particularmente devido à variabilidade observada na resposta individual à ERM $^{16}$.

A medida da ASTM, pela rinomanometria, também foi utilizada para avaliar os efeitos da ERM sobre a permeabilidade nasal ${ }^{37}$. Para tanto, comparou-se a ASTM de crianças de 10 a 14 anos com atresia maxilar antes da ERM e 1 ano após, utilizando como controle crianças sem atresia, da mesma faixa etária, avaliadas nos mesmos períodos. Após 1 ano, as crianças submetidas à ERM apresentaram um aumento estatisticamente significante na ASTM, o mesmo não ocorrendo para o grupo controle. Apesar dos resultados favoráveis, também neste caso salientou-se que a ERM não deveria ser realizada somente com finalidades respiratórias, visto que 30\% dos pacientes submetidos à ERM permaneceram com áreas seccionais inadequadas após o tratamento.

Nos últimos anos, a rinometria acústica passou a ser empregada para avaliar as dimensões internas da cavidade nasal. A técnica compreende a análise dos sons refletidos pela cavidade nasal em resposta a uma onda sonora incidente emitida por uma fonte acústica, os quais são captados por um microfone, ambos posicionados no interior de um tubo, adaptado a uma das narinas. As ondas são amplificadas e digitalizadas para análise. As áreas de secção transversa da cavidade nasal podem ser, então, observadas em um gráfico área - distância, a partir do qual também é possível calcular o volume de segmentos nasais específicos ${ }^{18,25,33}$ (Fig. 2).

A técnica permite medições consecutivas de diferentes segmentos da cavidade nasal, desde as narinas até as coanas e, desse modo, a identificação exata do local das diferentes constrições que contribuem para a resistência nasal, de forma rápida e não-invasiva, sem a necessidade da participação ativa do paciente, proporcionando informações 


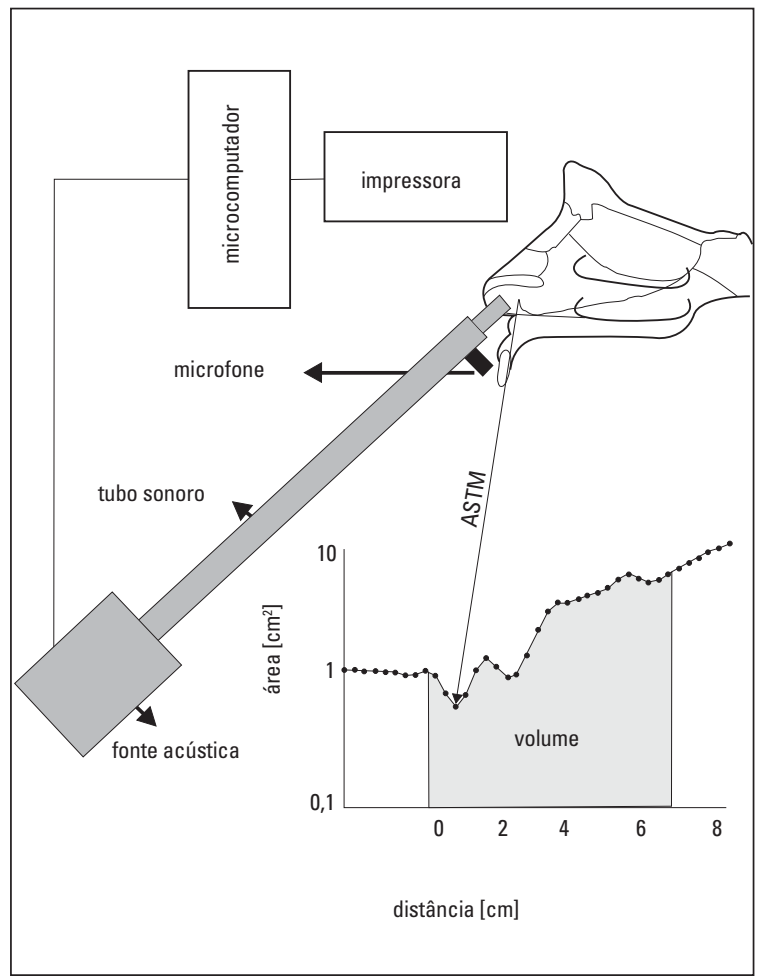

FIGURA 2 - Esquema representativo da rinometria acústica e gráfico área distância, com visualização do volume e da área de secção transversa mínima (ASTM) da cavidade nasal (Adaptado de BICAKCl et al. ${ }^{3}$ ).

topográficas sobre o perfil individual da cavidade nasal. Valores normativos de áreas e volumes nasais têm sido definidos ${ }^{12,33}$, assim como valores de vias aéreas obstruídas ${ }^{21,23}$.

Utilizando os recursos da rinometria acústica para avaliar os efeitos da ERM sobre a geometria nasal, estudos foram conduzidos, nos quais as áreas seccionais ${ }^{3,22}$ e volumes ${ }^{10,15}$ foram medidos antes da expansão $0^{3,10,15,22}$, após o período de ativação ${ }^{3,15,22}$, após período de contenção (90 dias após a expansão) $)^{3,15,22}$ e 1 ano após a expansão ${ }^{10}$. Todos utilizaram como amostra pacientes, com idade variando entre 10 e 12 anos, que possuíam maxila atrésica. Em um dos estudos ${ }^{15}$, volumes aferidos na região mais anterior da cavidade nasal aumentaram significativamente, em cerca de $10 \%$, na quase totalidade de casos analisados $(88,89 \%)$ após o período de contenção, não sendo constatada diferença entre os gêneros, nem variações sig- nificantes de ganho de volume após a expansão e após o período de contenção. Em estudo realizado no mesmo grupo de pacientes, mas publicado posteriormente ${ }^{22}$, resultados similares foram observados com relação à ASTM. Entretanto, em trabalho que avaliou o volume total da cavidade nasal ${ }^{10}$, aumentos estatisticamente significantes de volume foram observados 1 ano após o tratamento em crianças respiradoras bucais. Porém, constatou-se que apenas 8 das 14 crianças da amostra mudaram o padrão respiratório de bucal para nasal ${ }^{10}$. Em adição, a variação de ASTM mostrou certa relação com a época de realização da ERM, ou seja, maiores aumentos na ASTM foram constatados (após 3 meses de contenção) em pacientes que ainda não haviam atingido o pico de desenvolvimento na puberdade, em relação àqueles que já o haviam atingido ${ }^{3}$. A diferença, entretanto, não foi estatisticamente significante.

\section{DISCUSSÃO}

A associação entre a atresia maxilar e problemas respiratórios, particularmente a obstrução nasal, sempre foi assunto de interesse de clínicos e pesquisadores. Contudo, os conhecimentos atuais acerca das modificações estruturais determinadas pela ERM na cavidade nasal derivam, especialmente, de informações obtidas por meio de radiografias, que não fornecem uma visão tridimensional e nem permitem avaliar a cavidade nasal em seu aspecto funcional. A rinomanometria e a rinometria acústica são exames que preenchem as lacunas do exame radiográfico.

Dentre as pesquisas, publicadas na literatura, que avaliaram os efeitos da ERM sobre a função naso-respiratória por meio de rinomanometria e rinometria acústica, de maneira geral, parece haver consenso de que a ERM leva à modificação de parâmetros que expressam uma melhora nas condições de permeabilidade da cavidade nasal à passagem do fluxo respiratório.

Estudos que fizeram uso da rinomanometria como método de aferição demonstraram que, em 
parcela significativa dos pacientes, a ERM levou a uma diminuição da RN, estável ao longo do tempo ${ }^{16,17,24,29}$. Sabe-se que a resistência oferecida pelas fossas nasais à passagem do fluxo aéreo depende de seu tamanho, forma e comprimento ${ }^{30}$. Contudo, do ponto de vista fisiológico, a resistência depende, primariamente, da maior constrição (ASTM) da via aérea. No nariz normal de caucasianos, essa região corresponde à válvula nasal um orifício em forma de gota, situado logo após o vestíbulo nasal, que é delimitado pelo septo nasal, pela porção caudal da cartilagem lateral superior, pelo tecido fibro-adiposo que recobre a abertura piriforme e pelo assoalho nasal. A válvula nasal corresponde à região do trato respiratório que oferece maior resistência ao fluxo aéreo durante a respiração ${ }^{4}$. Muito provavelmente, a diminuição da RN após a ERM decorre do alargamento da válvula nasal, causado pela separação dos ossos maxilares promovida pelo aparelho expansor ${ }^{17,29}$. No aspecto funcional, isso significa mais espaço para passagem da corrente aérea.

É preciso, entretanto, analisar, com o devido cuidado, os resultados obtidos pelos autores que avaliaram a $\mathrm{RN}$. Isto porque essa variável é fluxodependente, ou seja, varia de acordo com o esforço respiratório. Um indivíduo pode ter, por exemplo, uma resistência nasal de $2 \mathrm{cmH}_{2} 0 / 1 / s$ quando o fluxo aéreo é igual a $300 \mathrm{ml} / \mathrm{s}$; de $2,5 \mathrm{cmH}_{2} \mathrm{O} / \mathrm{l} / \mathrm{s}$ num fluxo de $400 \mathrm{ml} / \mathrm{s}$; e de $3,5 \mathrm{cmH}_{2} \mathrm{O} / 1 / \mathrm{s}$ num fluxo de $500 \mathrm{ml} / \mathrm{s}$. Isto significa que as medidas da $\mathrm{RN}$ devem ser feitas em fluxos específicos, para que os resultados sejam comparáveis em momentos diferentes num mesmo paciente, entre grupos ou, ainda, entre diferentes estudos. Esta situação restringe a medida da $\mathrm{RN}$ como recurso metodológico, mas não invalida os estudos que dela fizeram uso.

Neste sentido, a medida da ASTM apresenta vantagens porque não é fluxo-dependente, ou seja, a medida da área nasal é a mesma em qualquer fluxo respiratório ${ }^{35}$. Utilizando esse recurso, foram comprovados os achados dos autores que mediram a RN, ou seja, também verificou-se um au- mento significativo da ASTM e, portanto, melhora da permeabilidade nasal ${ }^{37}$.

É importante enfatizar que não parece haver correlação significante entre a magnitude da expansão e as alterações observadas na resistência nasal ${ }^{29}$, visto que as alterações mais significativas puderam ser constatadas em pacientes com resistência nasal já elevada antes da expansão, provavelmente por obstrução nasal $16,17,29$.

Diferentemente da técnica rinomanométrica, a rinometria acústica é capaz de fornecer a localização exata de constrições esqueléticas e funcionais, assim como do volume, ao longo de toda a cavidade nasal. Com o advento desta nova técnica, novas informações foram obtidas com relação à geometria da cavidade nasal sob a ação da ERM. Aumentos significantes do volume do segmento anterior da cavidade nasal foram observados em pacientes analisados antes e após o uso de vasoconstritor, denotando variações de origem esquelética ${ }^{10,15}$. A magnitude dessa variação foi de cerca de 10\%, três meses após a expansão. Confirmando achados da rinomanometria, também se constatou variação significante da ASTM em parcela significativa de pacientes, da ordem de $16 \%{ }^{3,22}$. Uma tendência de maiores aumentos foi constatada antes do pico de crescimento na puberdade, o que pode estar relacionado à perda do efeito ortopédico da ERM, que se verifica com o aumento da idade ${ }^{24}$.

Há que se analisar com cuidado os achados da rinometria acústica. Estudos da geometria da cavidade nasal, comparando medidas realizadas por rinometria acústica e tomografia computadorizada, mostraram que na região mais anterior, até o nível das conchas nasais, as medidas obtidas com um e outro método não diferem entre si. Entretanto, constatou-se que as medidas de regiões mais posteriores são superestimadas pela rinometria acústica ${ }^{9,28}$, em outras palavras, a técnica perde em acurácia conforme as medidas se distanciam das narinas. Esta observação, em verdade, não invalida os achados dos estudos sobre os efeitos da ERM, uma vez que a porção mais anterior da cavidade 
nasal é a região mais atingida pela expansão.

Por fim, é importante destacar que - apesar das evidências de melhora da permeabilidade nasal que se seguem à ERM, por diminuição da RN e/ou aumento da ASTM e/ou aumento de volume - a literatura tem demonstrado que as alterações não são observadas em todos os pacientes e que, quando ocorrem, não são de magnitude suficiente para levar à alteração concomitante do modo respiratório de bucal para nasal. Em função disso, tornou-se consenso entre os autores que a realização da ERM não se justifica puramente com a finalidade de proporcionar melhora na função nasal de indivíduos com dificuldades respiratórias.

\section{CONCLUSÕES}

Em suma, após a revisão da literatura pertinente às repercussões da ERM sobre a permeabilidade das vias aéreas nasais, pode-se concluir que:
1) Estudos realizados com a rinomanometria demonstraram que a ERM determina uma diminuição significativa da resistência nasal em grande parte dos pacientes, assim como um aumento da área de secção transversa mínima nasal.

2) Estudos realizados com a rinometria acústica demonstraram que a ERM determina um aumento significativo do volume da cavidade nasal anterior, igualmente em parcela significativa dos casos, assim como da área de secção transversa mínima nasal.

3) Apesar dos evidentes benefícios trazidos pela ERM em termos de permeabilidade nasal, os estudos mostram que o procedimento não se justifica, por si, como forma de induzir um padrão nasal de respiração em respiradores bucais habituais.

\title{
The effects of rapid maxillary expansion on the nasal patency assessed by rhinomanometry and acoustic rhinometry
}

\begin{abstract}
The aim of this review was to analyze the findings reported in the literature about the effects of rapid maxillary expansion (RME) on nasal patency assessed by rhinomanometry, which measures the resistance offered by the nasal cavity to airflow, and acoustic rhinometry, which measures sectional areas and volumes of different segments of the nasal cavity. Studies using rhinomanometry have demonstrated a significant decrease of nasal resistance after RME on a significant number of patients, and also an increase in minimal nasal cross-sectional area. Studies using acoustic rhinometry have additionally shown a significant increase of anterior nasal volume. However, it is a consensus that RME is not justified only for the purpose of inducing a nasal pattern of breathing in oral breathers, despite the improvement of nasal patency caused by RME.
\end{abstract}

Key words: Palatal expansion technique. Acoustic rhinometry. Rhinomanometry.

\section{REFERÊNCIAS}

1. ANGELL, E. H. Treatment of irregularity of the permanent or adult teeth. Dent. Cosmos, Philadelphia, v. 1, no. 10, p. 540-544, May 1860.

2. ARAÚJO, A. M.; BUSCHANG, P. H. Conceitos atuais sobre o crescimento e desenvolvimento transversal dos maxilares e oportunidade de expansão mandibular. Rev. Dental Press Ortodon. Ortop. Facial, Maringá, v. 9, no. 3, p. 122-136, maio/jun. 2004.
3. BICAKCl, A. A. et al. Nasal airway changes due to rapid maxillary expansion timing. Angle Orthod., Appleton, v. 75, no. 1, p. 1-6, Jan. 2005.

4. BRIDGER, G. P. Physiology of the nasal valve. Arch.

Otolaryngol., Chicago, v. 92, no. 6, p. 543-553, Dec. 1970.

5. CAPELOZZA FILHO, L. Diagnóstico em Ortodontia. 1. ed. Maringá: Dental Press, 2000.

6. CAPELOZZA FILHO, L.; SILVA FILHO, O. G. Expansão rápida 
da maxila: considerações gerais e aplicação clínica. Parte I. Rev. Dental Press Ortodon. Ortop. Facial, Maringá, v. 2, n. 3, p. 88-102, maio/jun. 1997.

7. CAPELOZZA FILHO, L.; SILVA FILHO, O. G. Expansão rápida da maxila: considerações gerais e aplicação clínica. Parte II. Rev. Dental Press Ortodon. Ortop. Facial, Maringá, v. 2, n. 4, p. 86-108, jul./ago. 1997.

8. CAPELOZZA FILHO, L.; SUGUINO, R.; CARDOSO, M. A.; BERTOZ, F. A.; MENDONÇA, M. R.; CUOGHI, O. A. Tratamento ortodôntico da Classe III: revisando o método (ERM e tração) por meio de um caso clínico. Rev. Dental Press Ortodon. Ortop. Facial, Maringá, v. 7, n. 6, p. 99-119, nov./ dez. 2002

9. CAKMAK, O. et al. Value of acoustic rhinometry for measuring nasal valve area. Laryngoscope, Philadelphia, v. 113, no. 2 , p. 295-302, Feb. 2003.

10. CERONI COMPADRETTI, G. et al. Acoustic rhinometric measurements in children undergoing rapid maxillary expansion. Int. J. Pediatr. Otorhinolaryngol., Amsterdam, v. 70, no. 1, p. 27-34, Jan. 2006.

11. GALON, G. M.; CALCADA, F: URSI, W: QUEIROZ, G. V. ATTA, J.; ALMEIDA, G. A. Comparação cefalométrica entre os aparelhos de ERM bandado e colado com recobrimento oclusal. Rev. Dental Press Ortodon. Ortop. Facial, Maringá v. 8, n. 3, p. 49-59, maio/jun. 2003.

12. GOMES, A. O. C. Dimensões nasais e nasofaríngeas de indivíduos sem evidência de obstrução nasal avaliadas por rinometria acústica no repouso e na fala. 2004 Dissertação (Mestrado)-Hospital de Reabilitação de Anomalias Craniofaciais, Universidade de São Paulo, Bauru, 2004

13. HAAS, A. J. The treatment of maxillary deficiency by opening the midpalatal suture. Angle Orthod., Appleton, v. 35, no. 3, p. 200-217, July 1965

14. HAAS, A. J. Palatal expansion: just the beggining of dentofacial orthopedics. Am. J. Orthod., St. Louis, v. 57, no. 3 p. 219-255, Mar. 1970.

15. HAHN, L. et al. Avaliação do volume da cavidade nasal antes e após a expansão rápida da maxila por meio da rinometria acústica. Ortodon. Gauch., Porto Alegre, v. 3, n. 2, p. 85-96, dez 1999.

16. HARTGERINK, D. V.; VIG, P. S.; ABBOTT, D. W. The effect of rapid maxillary expansion on nasal airway resistance. Am. J. Orthod. Dentofacial Orthop., St. Louis, v. 92, no. 5. p. 381-389, Nov. 1987

17. HERSHEY, H. G.; STEWARD, B. L.; WARREN, D. W. Changes in nasal airway resistance associated with rapid maxillary expansion. Am. J. Orthod., St. Louis, v. 69, no. 3, p. 274-284 Mar. 1976.

18. HILBERG, O : JENSEN, F. T. PEDERSEN, O. F. Acoustic rhinometry: evaluation of nasal cavity geometry by acoustic reflection. J. Appl. Physiol., Bethesda, v. 66, no. 1, p. 295-303, Jan. 1989

19. LINDER-ARONSON, S. Respiratory function in relation to facial morphology and the dentition. Br. J. Orthod., London, v. 6 , no. 2, p. 59-71, Feb. 1979

20. LINDER-ARONSON, S.; LINDGREN, J. The skeletal and dental effects of rapid maxillary expansion. Br. J. Orthod., London, v. 6, no. 1, p. 25-29, Jan. 1979.

21. MAMIKOGLU, B.; HOUSER, S. M.; COREY, J. P. An interpretation method for objective assesment of nasal congestion with acoustic rhinometry. Laryngoscope Philadelphia, v. 112, no. 5, p. 926-929, May 2002.

22. MARCHIORO, E. M. et al. Efeito da expansão rápida da maxila na cavidade nasal avaliado por rinometria acústica. Rev. Dental Press Ortodon. Ortop. Facial, Maringá, v. 6, n. 1, p. 31-8, jan./fev. 2001

23. REBER, M.; RAHM, F.; MONNIER, P. H. The role of acoustic rhinometry in the pre- and postoperative evaluation of surgery for nasal obstruction. Rhinology, Utrecht, v. 36, no. 4, p. 184-187, Dec. 1998.
24. RIZZATO S. D. et al. Avaliação do efeito da expansão rápida da maxila na resistência nasal por rinomanometria ativa anterior em crianças. Ortodon. Gauch., Porto Alegre, v. 2, n. 2, p. 79-93, jul./dez. 1998.

25. ROITHMANN, R. et al. Acoustic rhinometry in the evaluation of nasal obstruction. Laryngoscope, Philadelphia, v. 105, no. 3 p. 275-281, Mar. 1995

26. SIQUEIRA, D. F.; ALMEIDA, R. R.; HENRIQUES, J. F. C. Estudo comparativo, por meio de análise cefalométrica em norma frontal, dos efeitos dentoesqueléticos produzidos por três tipos de expansores palatinos. Rev. Dental Press Ortodon. Ortop. Facial, Maringá, v. 7, n. 6, p. 27-47, nov./dez. 2002.

27. SILVA FILHO, O. G.; CAPELOZZA FILHO, L.; FORNAZARI, R. F.; CAVASSAN, A. O. Expansão rápida da maxila: um ensaio sobre a sua instabilidade. Rev. Dental Press Ortodon. Ortop. Facial, Maringá, v. 8, n. 1, p. 17-36, jan./fev. 2003

28. TERHEYDEN, H. et al. Acoustic rhinometry: validation by threedimensionally reconstructed computer tomographic scans. J. Appl. Physiol., Bethesda, v. 89, no. 3, p. 1013-1021, Sept. 2000.

29. TIMMS, D. J. The effect of rapid maxillary expansion on nasal airway resistance. Br. J. Orthod., London, v. 13, no. 4 p. 221-228, Oct. 1986

30. TRINDADE, I. E. K. Efeitos da cirurgia ortognática sobre a fala e a respiração de indivíduos com fissura lábio-palatina: análise acústica e aerodinâmica. 1999. Tese (Livre docência)Faculdade de Odontologia de Bauru, Universidade de São Paulo, Bauru, 1999.

31. TRINDADE, I. E. K.; TRINDADE JUNIOR, A. S. Rinomanometria nas estenoses craniofaciais. In: ZANINI, S. A. Cirurgia craniofacial: malformações. 1. ed. Rio de Janeiro: Revinter, 2000. cap. 12 , p. 119-126.

32. TRINDADE, I. E. K. et al. Dimensões das vias aéreas nasais na inspiração e na expiração. In: REUNIÃO ANUAL DA FEDERAÇÃO DE SOCIEDADES DE BIOLOGIA EXPERIMENTAL, 12., 1997, Caxambu. Livro de Resumos... Caxambu: [s.n.], 1997. p. 342-343

33. TRINDADE, I. E. K. et al. Volumes nasais de adultos aferidos por rinometria acústica. Rev. Bras. Otorrinolaringol., São Paulo, v. 73, n. 1, p. 32-39, jan./fev. 2007.

34. VIG, K. W. L. Nasal obstruction and facial growth: the strength of evidence for clinical assumptions. Am. J. Orthod. Dentofacial Orthop., St. Louis, v. 113, no. 6, p. 603-611, June 1998.

35. WARREN, D. W. A quantitative technique for assessing nasa airway impairment. Am. J. Orthod., St. Louis, v. 86, no. 4 p. 306-314, Oct. 1984

36. WARREN, D. W.; HAIRFIELD, W. M.; DALTSON, E. T. Effect of age on nasal cross-sectional area and respiratory mode in children. Laryngoscope, Philadelphia, v. 100, p. 89-93, Jan. 1990.

37. WARREN, D. et al. The nasal airway following maxillary expansion. Am. J. Orthod. Dentofacial Orthop., St. Louis, v. 91, no. 2, p. 111-116, Feb. 1987

38. WERTZ, R. A. Skeletal and dental changes accompanying rapid midpalatal suture oppening. Am. J. Orthod., St. Louis, v. 58 no. 1 , p. 41-66, July 1970

\section{Endereço para correspondência}

Inge Elly Kiemle Trindade

Departamento de Ciências Biológicas - FOB-USP

Alameda Octávio Pinheiro Brizola, 3-20

CEP: 17.093-900 - Bauru/SP

E-mail: ingetrin@usp.br 